\title{
A Research on Intelligent Classification of Urban Trash Bins Based on Machine Learning
}

\author{
Longyu Gao *, Zilong Liu, Luqi Shen, Songyun Shi, Yongzheng Lv \\ Tianjin University of Science and Technology, China; \\ E-mail:*ggafcc@163.com \\ www.tust.edu.cn
}

\begin{abstract}
Aiming at the problems of inaccurate, insensitive and general performance of the current intelligent garbage sorting bins, an intelligent garbage sorting bin based on machine vision is proposed. The trash can is mainly divided into five modules: main control module, machine vision module, classification module, overflow reminder module, and Wi-Fi Internet of Things module. The trash can uses convolutional neural networks to build an intelligent garbage classification model and classification algorithm to achieve rapid and accurate garbage classification. This experiment will be based on the identification of waste bottles, analyze the recognition characteristics of machine vision, and then propose methods to improve the accuracy of recognition.
\end{abstract}

Keywords: machine vision, convolutional neural network, intelligent classification

\section{Introduction}

At present, our country mainly relies on manual garbage classification, that is, placing several trash cans with different types of marks. Pedestrians need to judge which type of garbage they are in, and then put them in the correct trash can. Or hand over the trash to the staff nearby, and let them sort the trash and put it out. Although manual classification has achieved a certain effect, it is still not a long-term solution. This not only consumes a lot of time, manpower and material resources, but also loses the biggest advantage of the trash can itself, that is, convenience and time saving.

Therefore, the intelligent sorting trash can came into being. Although smart trash cans have long been proposed and designed, they are still not widely used in public places. The main reason is that the cost is too high and the garbage classification function is basically not available; and its main intelligent function is to use the infrared ranging module to sense whether the pedestrian is close to the trash can, and once it is close, the lid of the trash can is automatically opened for pedestrians to put Garbage; In addition, it also has voice prompts and driving functions. Obviously, this kind of smart trash can is mainly designed for family living, and many functions are not applicable in public relations situations.

For example, once a pedestrian just passes by the trash can and has no intention of throwing trash, the trash can will identify errors, consume unnecessary energy, and greatly increase costs. In addition, although there are some smart sorting trash cans on the market that can achieve recyclable and non-recyclable sorting functions, the use of sensor technology results in low classification accuracy and not widespread popularity.

\section{Overall design scheme of the system}

This article discusses an intelligent garbage sorting bin based on machine learning. The convolutional neural network is built through the artificial intelligence learning architecture, the training set is established in the early stage, the collected image data and image attribute tags are input, and the recognition model is obtained through multiple convolution operations; during image recognition, the garbage images collected by the camera are uploaded, After simple preprocessing, the 
identification model determines the attributes and categories of garbage ${ }^{1}$.

That is, according to recognition methods such as contour recognition, feature recognition, color recognition, and material recognition, find the common points between the two, and finally realize the judgment and classification. Finally, the corresponding signal is transmitted back to the single-chip microcomputer, and different steering gear angles are output according to the type of the returned signal, and then the flip board and the push rod are controlled to sort the garbage into the correct group. The network classification flowchart is shown in Figure 1.

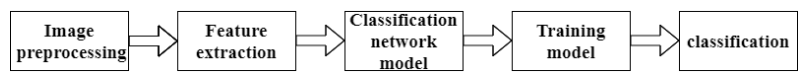

Fig.1. Classification process

The machine learning technology is applied to the system, and self-learning is carried out through the unsupervised learning task of clustering in machine learning. The specific work is: the system recognizes the characteristics of a large amount of garbage, and then presupposes. The unique characteristics of the four types of garbage are divided into four categories, each of which collects a large amount of data and saves the data in a database. When new garbage is encountered, the system extracts the data and matches it with the data in the database. Find out which type of data is the same or similar to the system, and classify it into one category. If there is no matching data in the system, the system automatically infers the new data from the existing data, and the system judges this by itself. Which category the new data belongs to, which makes the classification more accurate.

The main functions of the smart garbage sorting bin outlined in this article are: taking pictures of garbage thrown by pedestrians, intelligently sorting garbage, sensing whether the garbage bin is full, etc. It is mainly divided into five modules: main control module, machine vision module, mechanical module, sensor overflow module, Wi-Fi Internet of Things module. The main flow chart of the system operation is shown in Figure 2, and the structure design of the trash can is shown in Figure 3.

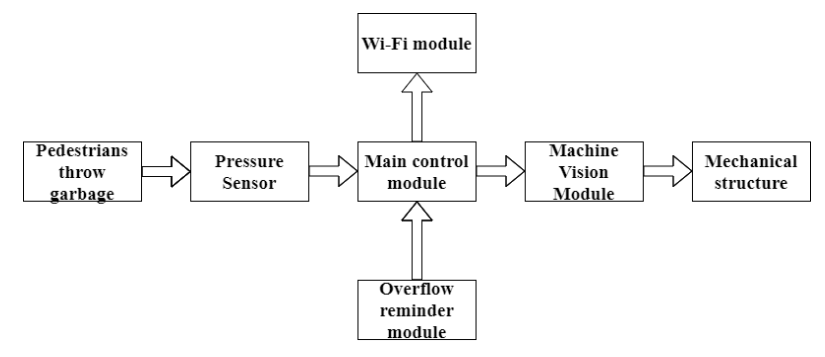

Fig.2. flow chart

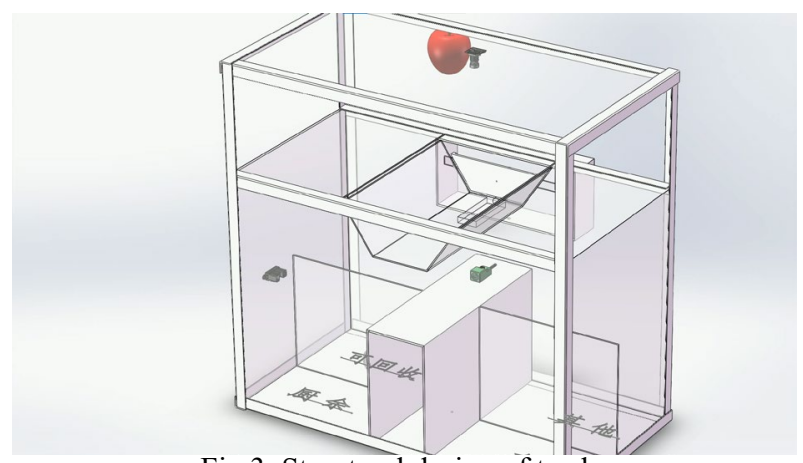

Fig.3. Structural design of trash can

In this experiment, a common beverage bottle is taken as an example. The beverage bottle data set comes from online and real-life photos. There are 4000 pictures in total, of which $60 \%$ of the training samples are 2400 ; the number of verification samples is $20 \%$, which is 800 ; testing The sample accounts for $20 \%$, which is 800 sheets. After completing the training of the garbage classification model, the model is tested for its recognition function and classification. According to the recognition results, the garbage classification model is optimized, and the collected garbage pictures are used as the input of the model, and the test is performed 800 times. Comparing the model output results with the real results, it is found that the recognition rate reaches $96 \%$. It can be seen that all bottles tested can be accurately identified, accurately and reliably. Later, based on this classification model, other garbage data training will be carried out.

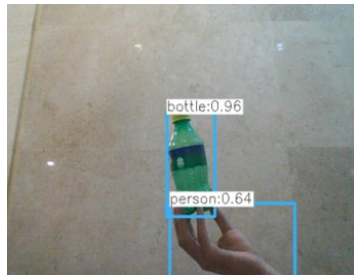

Fig.4. Recognition accuracy 


\section{Design of hardware control system}

\subsection{Main control module}

The main control module is mainly composed of STM32 controller. It is the brain of the entire system. When the pressure sensor senses that there is garbage being dropped, the STM32 controller controls the operation of the machine vision module. After the image recognition module recognizes the image category and feeds it back to the main controller, the STM controller will control the mechanical module to turn the corresponding angle to realize the intelligent classification and placement of garbage. The STM32 controller is shown in Figure 5.

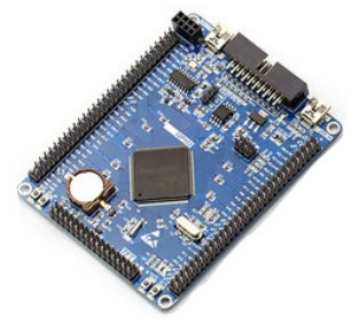

Fig.5. STM32F103ZET6

\subsection{Machine Vision Module}

The OpenMV module is installed at the garbage disposal port, and it captures images of garbage on the disposal platform by taking pictures ${ }^{3}$. It is mainly composed of a camera. The camera adopts a USB drive-free 1080P high-definition camera, which greatly improves the recognition resolution; at the same time, it also has a 160-degree wide angle, which greatly increases the shooting range; in addition, it also has a flash function to ensure that it takes pictures in a dim environment. OpenMV is shown in Figure 6.

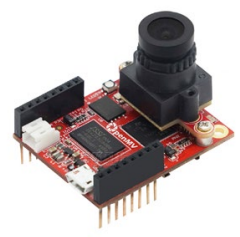

Fig.6. OpenMV

The machine vision module mainly analyzes and processes the photos taken by the machine vision module. The photos taken by the camera can be regarded as a large two-dimensional array. The elements of the array are called pixels, and their values are called gray values. The captured photos are converted into grayscale images, binary images and true color RGB images according to the size of the color and grayscale value, and the images are matched with the garbage training atlas pre-input to the convolutional neural network. According to the contour recognition, Recognition methods such as color recognition and material recognition seek common features between the two, and then identify the type of garbage that is thrown in according to the image algorithm written.

This module also adds the function of machine learning. First, it compares the various characteristics of the identified garbage with the existing characteristics of the system. If they are the same or similar, they are classified into one category and the data is stored in the system at the same time. Enlarge the system's database to make the next recognition more accurate and faster.

Compared with the current smart trash can, if there is nothing in common with the data stored in the system, the system will have problems that cannot be recognized.

For the system designed in this article, due to the addition of machine learning functions, when encountering such problems, the system will analyze its own existing data, and then self-judge the characteristics of the new data collected, thereby Perform accurate classification.

\subsection{Mechanical module}

After the image recognition module recognizes the type of garbage and feeds it back to the main control module, the main control module will control the steering gear to turn the corresponding angle according to the programmed algorithm.

Control the forward and backward movement of the front and rear push rods and the left and right flips of the left and right flaps to realize the movement of garbage in two dimensions. Finally realize the garbage classification to the corresponding trash can. The sorting mechanical structure is shown in Figure 7.



Fig. 7. Sorting mechanical structure

(C) The 2021 International Conference on Artificial Life and Robotics (ICAROB2021), January 21 to 24, 2021 


\subsection{Sensor overflow module}

This module is mainly used to detect whether the garbage in the trash can is full. In order to make the induction more accurate, it is divided into two small modules: weighing module and ultrasonic distance measuring module. The weighing module is used to sense whether the weight of the trash can exceeds the theoretical weight value. The weight value will be set according to the maximum allowable weight of different types of trash cans through investigation.

In order to prevent some special garbage thrown by pedestrians from being too heavy, causing the weight of the garbage can to exceed the theoretical weight value, but it is still not full, we also designed an ultrasonic ranging module at the mouth of the inner wall of the garbage can.

The working principle is that the ultrasonic transmitter emits ultrasonic waves in a certain direction, and starts timing at the same time as the transmitting time. The ultrasonic waves propagate in the air and return immediately when encountering obstacles on the way. The ultrasonic receiver stops timing immediately after receiving the reflected waves. Record the time $t$, then use the formula $S=v t / 2$ to calculate the distance between the ultrasonic source and the obstacle. When the garbage is not full, the actual distance measured is the inner wall diameter of the bucket; when the garbage is full, the actual distance measured is the distance from the ultrasonic transmitter to the garbage, so as to determine that the garbage can is full. The ultrasonic ranging sensor is shown in Figure 8.

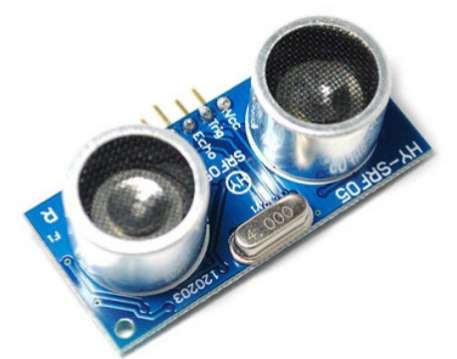

Fig.8. Ultrasonic distance sensor weight sensing and ultrasonic ranging is used to determine whether the trash can is saturated, which greatly improves the accuracy of the judgment. Compared with the existing smart garbage sorting bin, because of the use of machine learning functions, the recognition is more accurate and sensitive, and the practicability is higher. The recovery rate of recyclable garbage is greatly improved, and the loss of manpower, material resources and financial resources is reduced. , Has a certain use value. In general, the innovation of the system lies in simplifying the types of garbage classification and adding machine learning functions to improve the problem of garbage classification to the greatest benefit.

\section{References}

1. Golnabi, H., and A. Asadpour. Design and application of industrial machine vision systems. Robotics and Computer-Integrated Manufacturing, 2007, 23.6: 630-637.

2.Liu, Jianghai, Yadong Jiang. Design of intelligent trash can be based on machine vision. 2020 International Conference on Image, Video Processing and Artificial Intelligence. Vol. 11584. International Society for Optics and Photonics, 2020.

3.Vernon, David. Machine vision-Automated visual inspection and robot vision. STIA 92 (1991): 40499.

\section{Conclusion}

Through the design of software and hardware, this paper designs an intelligent sorting bin based on machine vision. It mainly uses image recognition methods to classify garbage. In addition, the dual combination method of 